\title{
La Legión Cóndor y la política exterior de la República de Bonn: relaciones germano-españolas durante el período de posguerra
}

\author{
URSULA HENNIGFELD \\ Heinrich-Heine-Universität Düsseldorf (Alemania) \\ orcid.org/0000-0002-0719-5520
}

Presentación: 24 en. 2019 | Aceptación: 11 jun. 2020 | Publicación: 31 oct. 2020

Cita recomendada: Hennigfeld, Ursula. 2020. «La Legión Cóndor y la política exterior de la República de Bonn: relaciones germano-españolas durante el período de posguerra». Dictatorships \& Democracies. Journal of History and Culture 8: 27-45. doi: https://dx.doi.org/10.7238/dd.voi8.3184

Resumen: El artículo se centra en los vínculos entre España y Alemania durante la Guerra Civil y en la posguerra: después de un breve resumen de la intervención de la Alemania nazi en la Guerra Civil, se describe la política exterior de la República de Bonn en relación con España para demostrar que la Alemania occidental recurrió a todo tipo de contactos de la época del Tercer Reich, tanto a nivel diplomático como económico o militar. La tercera parte se dedica, a modo de ejemplo, a los nexos entre la Legión Cóndor y la ciudad alemana de Düsseldorf a través de cinco figuras (W. Beumelburg, W. Bodden, J. von Richthofen, H. Schweizer y H. J. Herrmann).

Palabras clave: Legión Cóndor, Guerra Civil, Tercer Reich, política exterior de la República de Bonn

The Condor Legion and the Foreign Policy of the Bonn Republic: Germany-Spain Relations During the Postwar Period

Abstract: This article focuses on the connections between Spain and Germany during the Spanish Civil War and the postwar period. After providing a short summary of the German Nazi intervention in that war, the article describes the Bonn Republic's foreign policy towards Spain. It shows the manner in which contacts from the period of the Third Reich were used by West Germany on a diplomatic, economic and military level. The third part is dedicated to the ties between the Condor Legion and the city of Düsseldorf, based exemplarily on the life of five figures (W. Beumelburg, W. Bodden, J. von Richthofen, H. Schweizer, H. J. Herrmann).

Keywords: Condor Legion, Spanish Civil War, Third Reich, the Bonn Republic's foreign policy 
En el siguiente artículo quisiera ocuparme de los vínculos existentes entre la Guerra Civil, el nacionalsocialismo y — a modo de ejemplo — la historia de la ciudad de Düsseldorf. En la Universidad Heinrich Heine de Düsseldorf participo como hispanista en un grupo interdisciplinario de investigación que se ocupa del estudio de la etapa de la República de Bonn, en un marco tanto regional como internacional. Düsseldorf también fue el lugar de nuestro simposio internacional « ¿Ha terminado la Guerra Civil? España 1939-53», en el que participaron colegas de Alemania, España e Israel en 2019 y del que proceden las actas aquí reunidas. Düsseldorf ha de servir, por tanto, a título de ejemplo, pero naturalmente el caso podría ampliarse a otras ciudades.

En mi artículo me propongo poner de manifiesto algunos nexos entre Alemania y España en el período de posguerra y calibrar en qué medida este pasado común ha sido sometido a una reflexión crítica. Mi trabajo se inserta, así pues, en la investigación transcultural sobre procesos de memoria colectiva en ambos países. Mientras que en España la Guerra Civil representa hasta hoy en día una herida difícil de curar, en Alemania se ha olvidado casi por completo que el país participó en aquel conflicto de manera activa. En ambas sociedades de posguerra hay un elemento en común bastante significativo: incluso hasta mucho después del final de la Segunda Guerra Mundial, se negó la responsabilidad de la Legión Cóndor en la destrucción de la ciudad vasca de Guernica, tanto en la República Federal de Alemania como en la España franquista (Reig Tapia 2012, 909; Inal 2014, 105). Este ejemplo demuestra que la propaganda nazi y la franquista siguieron influyendo durante años en los discursos públicos y científicos. ${ }^{1}$ También se desmintió que submarinos alemanes estuvieran involucrados en la Guerra Civil. Hasta hoy se sigue negando (al menos la extrema derecha en Alemania, NPD) que se tratara de crímenes de guerra (NPD Bochum 2010; Oven 1978, 410). ${ }^{2}$

1 En su libro Spanien im Zweiten Weltkrieg, Ruhl (1975), por ejemplo, dedica varios capítulos a las filiaciones económico-militares y a la División Azul, pero las intervenciones de la Legión Cóndor en España se ocultan.

2 En el caso de Oven, no es nada sorprendente, ya que él mismo fue responsable de la sección de prensa de Goebbels y miembro de la Legión Cóndor. Véase Briesemeister $(2004,126)$. 
La intervención de la Alemania nazi en la Guerra Civil ha sido objeto de abundante investigación en España. En Alemania, por el contrario, este asunto se ha abordado mucho menos. Por eso presentaré brevemente, en el primer apartado, el estado actual de la investigación para ocuparme, en el segundo, de algunas interconexiones germano-españolas de posguerra seguramente poco conocidas. Con frecuencia, Alemania es considerada (también por parte de los españoles) como un caso modélico de Aufarbeitung, es decir, es visto como un país que aborda de forma crítica la historia del Tercer Reich con el objeto de plantear una política responsable y consciente de superación del pasado. Aunque este estereotipo (que es tanto heteroestereotipo como autorretrato) probablemente constituye un elemento distintivo de la identidad nacional alemana, quiero mostrar en lo que sigue que esa supuesta confrontación crítica modélica tiene ciertas lagunas sistemáticas. A esas continuidades y partes silenciadas de la historia común de ambos países se dedica la segunda parte, que aborda ciertos aspectos de la política exterior de la República de Bonn en relación con España. La tercera parte ilustra, a título de ejemplo, las trayectorias de cinco militares vinculados a Düsseldorf que, en razón de su incorporación a la Legión Cóndor, mantuvieron relaciones significativas con España.

\section{Intervenciones de la Alemania nazi en la Guerra Civil: un repaso de la historiografía alemana actual}

Es sabido que, ya antes de estallar la Guerra Civil, España era objeto de actividades bélicas y de espionaje organizadas por la Alemania nazi, por ejemplo, por parte de Wilhelm Canaris (Schüler-Springorum 2010, 61 y ss.). ${ }^{3}$ Posteriormente, se estima que Hitler envió, en apoyo a los golpistas y al futuro dictador español, unos 17.0oo soldados de la Marina, el Ejército y la Fuerza Aérea (Bernecker 1986). Contrariamente a lo que se suele creer en Alemania, no solo se produjeron operaciones de poca envergadura, en las que se experimentaba con el potencial bélico de la Fuerza

3 Para la política cultural de Alemania en España durante el período de entreguerras, véase De la Hera Martínez (2002). 
Aérea, sino que el apoyo recíproco entre los dictadores fascistas fue en aumento (Aschmann 1999).

Me limitaré a mencionar en lo que sigue tres ejemplos: la denominada Operación Fuego Mágico, la Operación Úrsula y la Legión Cóndor. La Operación Fuego Mágico (Unternehmen Feuerzauber) es quizás la intervención alemana más significativa en la Guerra Civil. Como es notorio, se trató de una maniobra en la que la Alemania nazi envió, en las primeras semanas de la guerra, aviones de carga a Marruecos para trasladar, estableciendo un puente aéreo, al Ejército africano de Franco a España. Entre julio y agosto de 1936, los Junker Ju 52 transportaron desde el protectorado español hasta la península un contingente de alrededor de 13.000 combatientes entre legionarios y regulares, soldados marroquíes al servicio del Ejército español (Whealey 1989).

Pero no se emplearon solo aviones para apoyar la política de Franco: la Marina alemana estuvo asimismo al servicio de los sublevados. Esta intervención, que en un principio fue secreta, se conoció bajo el código de «Operación Úrsula»; Úrsula era el nombre de la hija del comandante en jefe de la Marina alemana, Karl Dönitz (Herzog 1991, 5). Las fuentes relativas a esta cuestión que han pervivido son insuficientes, dado que la mayoría de documentos fueron eliminados y los implicados juraron por escrito guardar silencio, un voto al que aparentemente todavía se sentían sujetos durante la posguerra. Según las investigaciones de Bodo Herzog (1991, 2) para el semanal Die Zeit, entre 1936 y 1939 se hizo uso de doce torpederos alemanes, seis cruceros ligeros, tres acorazados y dos submarinos. Se calcula que, en total, se trasladó a España al menos una cuarta parte de la fuerza naval existente (Deutsches U-Boot-Museum, s. f.).

Muchos comandantes que posteriormente participarían en la Segunda Guerra Mundial estuvieron ejercitándose en operaciones con submarinos en la Guerra Civil. Era entonces comandante en jefe de la Marina alemana en España el contralmirante Hermann Boehm. En 1936 se enviaron en misión secreta dos submarinos a las costas aún controladas por los republicanos en España. La misión se describió oficialmente como unas simples maniobras de entrenamiento. No obstante, en esa ocasión fueron atacadas las fuerzas navales republicanas. Para los submarinos se utilizaron sobrenombres: los U-33 y U-34 se convirtieron en Tritón y Poseidón (Herzog 
1991, 6). Los magros resultados fueron más bien un motivo de deshonra para la Marina, al menos desde la perspectiva de la cúpula militar alemana. Por este motivo, el ministro de la Guerra y comandante en jefe de las fuerzas armadas alemanas, el mariscal de campo Werner von Blomberg, ordenó detener la Operación Úrsula el 10 de diciembre de 1936. Pese a ello, dos días después de la resolución el submarino U-34 atacó y hundió un submarino republicano (Herzog 1991, 6). Oficialmente, la intervención de la Marina alemana concluyó a finales de 1938. Pero fue el 6 de junio de 1939 cuando Hitler recibió a los combatientes alemanes en España en la Cancillería. En el desfile triunfal de las tropas combatientes en España sonaron la Marcha de los bombarderos de la Legión Cóndor (en alemán, Bombenfliegermarsch der Legion Condor, con música de Hans Teichmann y texto del teniente Erich Schlecht) y la canción Surcamos el vasto mar (en alemán, Wir zogen übers weite Meer, con música de Wolfram Philipps y texto de Christian Jährig). Entre esas tropas se encontraba, por ejemplo, el lugarteniente y comandante de submarino Harald Grosse, condecorado por Hitler con la Cruz Española de Oro (Herzog 1991, 14).

La Operación Úrsula se puede calificar de guerra submarina ilícita contra la República Española. Hasta la fecha, estas operaciones militares no han sido suficientemente estudiadas por la historiografía oficial de la Marina alemana. Bodo Herzog escribió a propósito de esto en Die Zeit:

La historiografía de la Marina alemana ha eludido deliberadamente este tema, y autores de narrativa memorialista han ocultado o negado la verdad. El hundimiento del submarino español C-3 - antes del ataque terrorista en Guernica - fue el primer crimen de guerra de las fuerzas armadas alemanas, un acto vulgar y brutal, que en términos jurídicos de conflicto - y con arreglo al lenguaje de entonces - se tendría que llamar «piratería».4

El libro del comandante de submarino Günther Prien (Mein Weg nach Scapa Flow, 1940, con 900.00o ejemplares vendidos) y del capitán de corbeta Fritz Otto Busch (Narvik, 1940, con 600.00o ejemplares vendidos) se contaron entre los libros más leídos por los jóvenes en el Tercer

4 Todas las traducciones de fragmentos en alemán que se citan en este artículo son mías. 
Reich y demuestran el esfuerzo propagandístico para alimentar la fascinación por la Marina (Adam 2010, 150 y ss.). La propaganda nazi y, más en particular, los libros para jóvenes sobre la Legión Cóndor o actividades como la Semana Española (organizada en 1938 en Fráncfort) tuvieron como efecto un auge considerable del aprendizaje del español en los institutos de enseñanza media (Hausmann 2001, 218). El comandante de submarino Kurt Freiwald, que ordenó la destrucción del submarino republicano, reanudó su carrera en la Marina alemana en los años cincuenta (Hausmann 2001, 218).

La ofensiva más conocida es ciertamente el bombardeo de la villa de Guernica el 26 de abril de 1937 por parte de la Legión Cóndor y la Aviación Legionaria italiana. ${ }^{5}$ No fue solo Franco quien se alegró del supuesto éxito de la destrucción de Guernica; también Hermann Göring comprobó con satisfacción, a propósito de este cruento episodio bélico, que la Guerra Civil podía ser un útil banco de pruebas para el cuerpo de aviación militar alemán. Y no solo eso: en compensación, Hitler obtuvo de Franco el permiso de extracción de minerales en el País Vasco. A partir de entonces, la Alemania nazi pudo adquirir materias primas por debajo del precio del mercado mundial. Así, la Sociedad Hispano-Marroquí de Transportes (HISMA), empresa fantasma del nacionalsocialismo, adquirió en 1937 los derechos sobre minas de hierro, plomo, wolframio, estaño, zinc, cobalto y níquel (Bernecker 1986, 45-46; Aschmann 1999, 32). Desde ese momento, cada vez que España quería establecer vínculos económicos con otras naciones, debía informar previamente a la Alemania nazi; en caso contrario, podía ser retirada la Legión Cóndor.

El jefe del Estado Mayor, Wolfram von Richthofen, asumió la dirección de los combates aéreos hasta el año 1943 y murió en 1945, prisionero de guerra de los americanos. Concluida la Segunda Guerra Mundial, el primer comandante de la Legión Cóndor, Hugo Sperrle, fue absuelto de to-

5 Esta perspectiva es problemática por su reduccionismo, como señala Joan Maria Thomàs: «Other tragedies, however, are sometimes obscured by circumstances elsewhere. This is the case of the "other Guernicas": that is to say, the bombings of Otxandio, Durango and Elorrio in Biscay" (Thomàs 2019, 7), y aún podrían aducirse los casos de Madrid, Barcelona, Málaga, Almería, Alicante y otras muchas ciudades y pueblos. No obstante, me ceñiré al ejemplo de Guernica para revelar las huellas de la Guerra Civil que llegan a Düsseldorf. 
dos los cargos en los Juicios de Núremberg. Muchos otros reingresaron en el Ejército de la República Federal de Alemania (RFA) después de la guerra. Por lo demás, conviene tener bien presente las palabras de Stefanie Schüler-Springorum, que afirma que la imagen mediática de España que había ofrecido la Alemania nacionalsocialista permanecería prácticamente intacta en los años cincuenta y sesenta, y que la interpretación nacionalsocialista sobre la guerra y los combates aéreos estaría presente y se podría constatar en la prensa y ediciones de libros desde el período de la República de Bonn hasta los años setena (Schüler-Springorum 2010, 252; Lehmann 2006, 37). ${ }^{6}$

Alemania no se disculpó oficialmente por el ataque aéreo a Guernica hasta 1997. El municipio de Guernica solicita desde hace décadas, entre tanto, una comisión de investigación germano-española, a la que debería facilitarse cualquier documento que sirviera para el esclarecimiento de los hechos. Según tengo entendido, esta petición todavía no ha sido atendida (Bernecker 1986, 43). ${ }^{7}$

\section{Políticas de intervención alemana en relación con España en la República de Bonn ${ }^{8}$}

Después de la Segunda Guerra Mundial, tanto a España como a la República de Bonn las seguía uniendo su postura anticomunista. El miedo del Gobierno de Adenauer al bolchevismo motivó el restablecimiento de una relación de cooperación con España. En los años inmediatamente

6 Es tanta la fascinación y el carácter emblemático de la aviación que la propaganda nazi hizo uso de ella a través de películas como El triunfo de la voluntad (Triumph des Willens, 1934, Leni Riefenstahl), en la que Hitler desciende como del cielo ante las masas, es decir, que aterriza en el congreso del Partido Nacionalsocialista en Núremberg. Pero en la literatura también se extendió el uso del mito del héroe aviador: en novelas y autobiografías se estableció el prototipo de un intrépido, apuesto y vigoroso caballero de los aires (véase Schüler-Springorum 2010, 38).

7 Para la repercusión internacional del bombardeo de Guernica y su presencia mítica en discursos actuales, véase Chihaia \& Hennigfeld (2020).

8 La información que expongo a continuación está basada fundamentalmente en la monografía de Walter Lehmann Die Bundesrepublik und Franco-Spanien in den $50 e r$ Jahren («La República Federal Alemana y la España franquista de los años cincuenta»), publicada en 2006, y en la de Birgit Aschmann «Treue Freunde...»? Westdeutschland und Spanien 1945-1963 (« ¿Amigos fieles? La Alemania del Oeste y España, 1945-1963»), publicada en 1999. 
posteriores a la guerra, España se encontró políticamente aislada, pues la constitución del régimen de Franco solo había sido posible años atrás gracias a la colaboración de la Alemania nacionalsocialista y la Italia fascista. Los países de Europa occidental fueron recuperando la democracia tras la Segunda Guerra Mundial, a excepción de los dos estados de la península ibérica. En cualquier caso, a España se la consideraba entonces como un remanente indeseable de la era fascista. En la Conferencia de Potsdam, Stalin se mostró a favor de suspender todo contacto con la España de Franco. En 1946 se inició un boicot, organizado desde la ONU, para retirar de Madrid a los representantes diplomáticos de casi todos los países. Solo Argentina, Portugal y el Vaticano consintieron que sus embajadores permanecieran en España (Lehmann 2006).

Las políticas de la Alemania occidental en relación con España entre los años 1949 y 1960 se vieron influidas por la forma en la que los integrantes de la Legión Cóndor y la División Azul reclamaban sus derechos de pensión. En 1960 se incorporó la División Azul — formada, como es sabido, por voluntarios españoles que lucharon contra la Unión Soviética bajo mando alemán - a la Asociación de Soldados Alemanes (Aschmann 1999, 450). Después de todo, esta unidad de infantería estuvo en el frente oriental al servicio del Ejército alemán para combatir el bolchevismo. Los alemanes debían estar agradecidos por el apoyo militar, según expresó el agregado militar Achim Oster en 1960. ${ }^{9}$

Asimismo, desde los años cincuenta se produjo un acalorado debate acerca de cómo calcular el período de servicio de los voluntarios de la Legión Cóndor. En 1954, aunque los ataques de la Legión Cóndor se consideraban violaciones del derecho internacional, los militares encuadrados en ella quedaron exonerados de toda culpa, alegando obediencia debida. Se consideró que la disputa en torno a la naturaleza de la intervención de la Legión Cóndor no debía perjudicar a los soldados implicados. Y fue así como el paso por la Legión Cóndor terminó considerándose válido, a efectos de cómputo de la pensión, como período de servicio militar a escala nacional (Lehmann 2006, 176 y ss.).

9 Hans Karl Joachim Achim Oster era hijo de Hans Oster, un estrecho colaborador de Wilhelm Canaris (Aschmann 1999, 354).

DICTATORSHIPS \& DEMOCRACIES 8 (2020) - E-ISSN: 2564-8829 · PUNCTUM, UNIVERSITAT OBERTA DE CATALUNYA \& FUNDACIÓ CARLES PII SUNYER 
En aquellos años se discutió, además, si los supervivientes españoles de los campos de concentración o sus familiares debían ser indemnizados. La decisión final fue la de negar las indemnizaciones para no poner en peligro las buenas relaciones con el Gobierno español, siendo aquellos adversarios de Franco. A diferencia de otros países europeos, España renunció entonces a un convenio de indemnización de la República Federal de Alemania. Y tampoco tuvo lugar ningún debate profundo sobre los alemanes que lucharon en ambos bandos en la Guerra Civil. De hecho, apenas se habló de los casi 3.000 alemanes antifascistas que combatieron en las Brigadas Internacionales del lado de los republicanos. Ninguno de ellos recibió prestaciones estatales por su servicio militar porque se suponía de forma generalizada su vinculación al comunismo. Hasta 1972 no se modificó a este respecto la legislación para la asistencia a las víctimas de guerra; a partir de entonces, se reconoció el derecho a solicitar compensaciones (Lehmann 2006, 176 y ss.).

A nivel diplomático, se restablecieron muchos de los contactos personales ya existentes antes de 1945 entre alemanes y franquistas. Muy pocos políticos alemanes de la década de los cincuenta compartían una visión antifranquista de la Guerra Civil; no era conveniente adoptar una perspectiva prorrepublicana, porque ese enfoque resultaría más bien afín al defendido por la República Democrática Alemana (RDA). En cambio, cada vez que se mencionaba la barbarie bolchevique se utilizaban el tono y los argumentos de la propaganda nacionalsocialista. Lehmann, entre otros, habla acertadamente de paradigmas que sobrevivieron a la época del nacionalsocialismo, y lo ejemplifica con el fragmento de un discurso del ministro de Asuntos Exteriores, Walter Hallstein, de 1952:

Al comienzo de la Guerra Civil española, en 1936, fueron los aviones alemanes de transporte los que llevaron las tropas africanas de Franco a la península, haciendo una contribución no menos importante que la de los voluntarios de la Legión Cóndor al éxito del levantamiento nacional. Esta ayuda militar no la ha olvidado el pueblo español. (Lehmann 2006, 37) 
Según indica Lehmann (2006, 36), en la República de Bonn de los años cuarenta y cincuenta no se suscitó ningún debate público sobre la imagen de España ofrecida por la propaganda nacionalsocialista. ${ }^{10}$

En cuanto al aspecto económico, cabe mencionar que en 1948, con arreglo a los Acuerdos de Madrid, fueron expropiados los bienes y activos alemanes en España: el Estado español tomó posesión de empresas, patentes y marcas comerciales, entre otros. Se trataba, sobre todo, de bienes y activos relacionados con la industria del sector químico (IG Farben) y eléctrico (AEG, Siemens, Osram) (Lehmann 2006, Aschmann 1999, Bernecker 1986). Pero al poco tiempo la mayoría de estos bienes y activos volvió a estar en manos de entidades alemanas (sobre todo de AEG y las empresas sucesoras de IG Farben), que adquirieron de nuevo, de forma no oficial, sucursales y participaciones en dichos negocios.

Desde los años cincuenta se constató una relación de reciprocidad, en la medida en que la República de Bonn contrataba a obreros inmigrantes españoles y España se convertía en el nuevo destino turístico de no pocos alemanes. Pero también hubo en esa década otra forma de cooperación, a saber, la de carácter militar. El ex teniente general, experto en armamento, Erich Schneider actuó como asesor del entonces ministro del Ejército, Agustín Muñoz Grandes, que había sido comandante de la División Azul y a quien se había concedido la Cruz de Caballero (Aschmann 1999, 52). El propósito era modernizar la industria armamentística con ayuda de especialistas alemanes. Schneider vio en España el lugar ideal para el desarrollo y la producción de armamento de la República de Bonn, soslayando así las restricciones impuestas a Alemania por parte de los Aliados. La empresa Rheinmetall de Düsseldorf se fijó como empresa organizadora e implementadora de proyectos, disposición a la que se renunciaría posteriormente.

En la misma década de los cincuenta, la aviación alemana aprovechó la oportunidad para trabajar de nuevo en el área de desarrollo de aeronaves, eludiendo las prohibiciones de los Aliados. Constructores y empresarios

10 Véase también Aschmann (1999, 332-333): «Podemos resumir que la República de Bonn apuesta a todos los niveles por una relación de amistad con el régimen de Franco. [...] Esto se manifiesta sobre todo con respecto a las relaciones militares». 
del sector aeronáutico como Willy Messerschmitt y Claude Dornier, que en los años treinta habían trabajado exitosamente en los programas nacionalsocialistas de producción de armamento, se establecieron entonces en España para desarrollar sus proyectos (Lehmann 2006, 149 y ss.; Rebok 2010, 371; Aschmann 1999, 335-336). Dornier dirigió en Madrid, de 1950 a 1955, una empresa constructora; Messerschmitt trabajó para el Ministerio del Aire español —encargado de la aviación civil y militar - como consultor de la compañía Hispano Aviación en Sevilla (Rebok 2010, 373; Aschmann 1999, 336-341).

Con respecto a las conversaciones exploratorias de los alemanes en Madrid, surgidas de la intención de posibilitar la formación y el entrenamiento de las fuerzas aéreas alemanas, Lehmann se expresa justificadamente con desaprobación: «Aviones sobre España: una imagen que puede haber sido militarmente plausible, pero que solo se puede interpretar como una expresión de ignorancia histórica en relación con la intervención de la Legión Cóndor en la Guerra Civil española» (Lehmann 2006, 265).

El historiador Carlos Collado Seidel ha señalado que, desde el año de la fundación de la República de Bonn, las élites políticas y sociales insistieron, en el caso concreto alemán, en abandonar las llamadas «medidas de depuración» y reiniciaron sus actividades sin plantear recriminación alguna. ${ }^{11}$ Esto también es visible en la rápida disminución de los casos penales pendientes. Por otra parte, las condenas fueron muy pocas; en lugar de ello, se propugnaron más bien la amnistía y la liberación de presos. Incluso en el tercer juicio del denominado «proceso Majdanek» (en contra de los encargados del campo de concentración del mismo nombre) se emitieron sentencias relativamente benevolentes. Los procesos de desnazificación permitieron que, para abril de 1951, los funcionarios del Tercer Reich pudieran estar ya rehabilitados y haber recuperado su empleo al servicio del Estado. Es conocido el modo en el que Konrad Adenauer respondió ante las críticas por tener un Ministerio de Relaciones Exteriores ocupado por antiguos nazis: «El que no tiene agua limpia, que no derrame la sucia»

11 Véase también Rebok (2010, 379): «El estrecho intercambio establecido entre las dos dictaduras entre 1939 y 1945 conoció en los años siguientes vías de prolongación asentadas en las redes de relaciones personales entre científicos, técnicos y académicos de los dos países.» 
(Collado Seidel 2012, 90). Las políticas de reintegración de exfuncionarios nazis coinciden temporalmente con la aprobación de la Resolución de indemnización por agravios del nacionalsocialismo a miembros de la Administración pública (Gesetz zur Regelung der Wiedergutmachung nationalsozialistischen Unrechts für Angehörige des öffentlichen Dienstes). En cambio, el acuerdo para compensar a las víctimas de la Shoah fue bastante controvertido y aprobado en el Parlamento por una estrecha mayoría. Aschmann $(1999,14)$ deduce que «el modo de relacionarse de la joven democracia germano-occidental con la España franquista deja entrever las convicciones con respecto al propio pasado alemán». ${ }^{12}$ Bernecker (2009, 72) tiene una visión bastante similar de la justicia transicional española: «Hasta el final del Gobierno de Aznar, el ejecutivo obstaculizaba prácticamente cualquier trabajo acerca de la memoria histórica al considerar que implicaba una condena de los crímenes franquistas».

\section{Entre Guernica y Düsseldorf: cinco trayectorias}

Existen varios nexos entre algunos miembros de la Legión Cóndor y la historia de la ciudad alemana de Düsseldorf en el Tercer Reich. A sabiendas de ello, revisé sistemáticamente las listas de miembros de la Legión Cóndor y, a pesar de la dificultad relativa a las fuentes, pude rastrear algunas huellas y concretar algunos datos.

Según la información que obtuve, hay dos personas vinculadas a Düsseldorf que participaron en el bombardeo de Guernica como miembros de la Legión Cóndor. La primera de ellas es Werner Beumelburg, quien, nacido en Traben-Trarbach, combatió en la Primera Guerra Mundial, estudió luego ciencias sociales y ejerció posteriormente como corresponsal del Deutschen Soldaten Zeitung («Periódico de los Soldados Alemanes») del Ministerio de Defensa del Reich en Berlín (Schmigalle 1986, 207-208). Entre 1924 y 1926 trabajó como redactor para el periódico Düsseldorfer Nachrichten («Noticias de Düsseldorf»). Este comandante de la Fuerza Aérea

12 Para una comparación germano-española de la memoria de la Guerra Civil en la literatura, la fotografía y el cine, véase Bannasch et al. (2005). La memoria histórica de ambos países las comparan también Olmos et al. (2009). 
tuvo bastante predicamento como autor durante la dictadura nacionalsocialista (Adam 2010, 140-141), ${ }^{13}$ lo cual se comprueba, por ejemplo, en su participación en la Academia Alemana de Literatura (Deutsche Akademie der Dichtung) y en la escritura de diversos documentos propagandísticos. En 1939, por encargo del ministro de Aviación, publicó el libro Kampf um Spanien. Die Geschichte der Legion Condor («La lucha por España. La historia de la Legión Cóndor»), que para 1942 contaba ya unos 70.000 ejemplares vendidos (Schmigalle 1986, 207). En este libro escribe que la Legión Cóndor «ha sido, por el espíritu y la capacidad de su armamento, un testimonio de satisfacción y tranquilidad para los alemanes y sus aliados; y a su vez, una advertencia y una lección para nuestros enemigos» (citado en Schmigalle 1986, 207). Beumelburg no se retractó de su opinión después de la Segunda Guerra Mundial. Por el contrario, en el período de la posguerra siguió publicando libros dedicados a su tema favorito: los combates aéreos. Su Kampf um Spanien de 1939 se reeditó, sin revisión alguna, en una fecha tan avanzada como el año 1980 (Briesemeister 2004, 126).

El segundo personaje es Wilhelm Bodden, teniente de la Legión Cóndor, del que se ha hallado poca información fiable. En las listas de muertos y desaparecidos de la Legión Cóndor se incluyen algunos datos sobre él: por el hecho de que sus padres vivían en Düsseldorf, se puede suponer que también él residía en esta ciudad antes de la guerra. En 1939 fue una de las veintiocho personas que recibieron la Cruz Española de Oro con Diamantes por su coraje y valentía (véase Lexikon der Wehrmacht, s. f.). Hugo Sperrle y Wolfram von Richthofen recibieron la misma condecoración. La muerte de Bodden el 24 de febrero de 1939, en un accidente aéreo cerca de Niza, conllevó que dicho galardón fuera concedido a título póstumo y enviado a Düsseldorf al domicilio de sus padres.

El otro grupo de personas a las que me referiré a continuación se establecieron en Düsseldorf después de su intervención en la Legión Cón-

13 Adam (2010, 140-141) opina que Beumelburg figura - junto con Edwin Erich Dwinger, Hans Zöberlein o Ernst Jünger- entre los autores más leídos en el período de entreguerras. Escribe esencialmente sobre las batallas de la Primera Guerra Mundial, sugiriendo que, por respeto a los muertos, se debería perseverar en su misma causa, aun a costa de futuros sacrificios bélicos. Entre sus libros más vendidos están la crónica Sperrfeuer um Deutschland (1929, con 363.00o ejemplares vendidos) y la novela Die Gruppe Bosemüller (1930, con 164.000 ejemplares vendidos). 
dor. Joachim von Richthofen II fue constructor de aviones. Después de la guerra se mudó a Düsseldorf, donde falleció en 1981. En la Guerra Civil analizó para la Legión Cóndor el impacto de los bombardeos: informó a sus superiores con detalle acerca de la manera más efectiva de destruir poblaciones españolas. En 2003 Per Hinrichs escribió sobre esa cuestión en la revista Spiegel afirmando que Von Richthofen estudió minuciosamente el ataque a Guernica (Hinrichs 2003). Según los reportes de Von Richthofen, el bombardeo fue una operación pensada para desarrollarse en varias fases. En primera instancia, se arrojaron bombas que provocaron un gran número de incendios en la techumbre de las casas. Luego siguieron los ataques con bombas de 250 kilos de material explosivo, con las que se destruyeron las conducciones de agua para frustrar, así, la posibilidad de cualquier tentativa de extinción del fuego (Hinrichs 2003, 34). No se alcanzó un éxito total, según Von Richthofen, por el hecho de que algunas bombas cayeron, lamentablemente (como él mismo se ocupó de recalcar), en lugares deshabitados. Los resultados fueron expuestos con detallismo técnico y sin un ápice de compasión: «El nivel de destrucción alcanza un $75 \%$ de la población, haciendo uso de $31.000 \mathrm{~kg}$ de explosivos arrojados desde una altura de entre 600 y $800 \mathrm{~m}$ (Hinrichs 2003, 34).

Heinz Schweizer fue un artificiero registrado en las listas de la Legión. Luego de su servicio en la Guerra Civil, se convirtió en comandante encargado de la brigada de explosivos del distrito de Kalkum en Düsseldorf, a comienzos de la década de 1940. Schweizer adquirió gran fama en el Tercer Reich por concebir nuevas técnicas de desactivación de artefactos explosivos no detonados, otorgándosele el apelativo de Der Mann mit den Stahlnerven («el hombre de nervios de acero») en la propaganda nacionalsocialista. En 1943 fue honrado con la Cruz de Caballero (Ritterkreuz) como primer militar de la Fuerza Aérea no aviador (Henkel 2016, 37). Heinz Schweizer es un personaje bastante ambiguo: por un lado, en ambientes militares estaba considerado como un héroe nacionalsocialista; por otro, era conocido por haber tratado bien a los prisioneros y por haber salvado de la Gestapo a varios de ellos al final de la guerra. Después de un breve período como prisionero de guerra, regresó a su casa familiar, donde murió en 1946, asesinado por soldados rusos ebrios que se disponían a saquear la vivienda (Henkel 2016,37). Algunos de sus métodos aún se usan 
para la desactivación de material bélico de la Segunda Guerra Mundial. En 2004 se publicó el libro Hauptmann (W) und Ritterkreuzträger Heinz Schweizer. Feuerwerker und Sprengkommandoführer («Heinz Schweizer, comandante y Cruz de Caballero. Artificiero y comandante de la brigada de explosivos»), de Heinz Thamm. El autor es, asimismo, experto en bombas y excombatiente del Ejército alemán. Según afirma, se trata de un análisis histórico, si bien incurre en halagos a Schweizer absolutamente acríticos. En su libro se encuentran, por si fuera poco, anuncios publicitarios de fabricantes de armas. Los beneficios recaudados por los anuncios y las donaciones se habían de destinar, según el autor, al mantenimiento de la tumba de Heinz Schweizer.

El último exponente, Hans-Joachim Herrmann, no fue un miembro cualquiera de la Legión Cóndor. Herrmann fue nada menos que el celebrado héroe aviador del Tercer Reich que hundió doce barcos y derribó nueve bombarderos. Fue, además, el inventor de lo que se denominó en la prensa de la posguerra la «táctica del combate aéreo radical», a la que los nazis y el mismo Herrmann llamaron la Wilde-Sau-Taktik («la táctica del jabalí») (Gunkel 2009; Irving 1965, 120), un procedimiento puesto en práctica en el año 1943 que consistía en atacar aviones de los Aliados por la noche y sin radar, y apuntando con reflectores al enemigo para que los aviones de caza de Herrmann pudieran hacer blanco en ellos (Gunkel 2009; Irving 1952, 120).

Cuando ya se vislumbraba la derrota de los alemanes, Herrmann desarrolló otro controvertido plan, todavía más drástico: se propuso llevar a cabo ataques aéreos con pilotos kamikazes. En la base aérea militar de Stendal, se radicalizó a jóvenes pilotos y se les preparó para su última misión con propaganda, chocolate, coñac, vino y cigarrillos. El 7 de abril de 1945 se consumó su monstruoso plan: un escuadrón de 183 aviones de combate ascendió a 11.000 metros de altitud y esperó a su adversario. La consigna Alle Geier und Falken - Angriff frei! («Buitres y halcones, jal ataque!») (Gunkel 2009) era la contraseña para lanzarse en picado sobre ellos a 800 kilómetros por hora. Desde el punto de vista nacionalsocialista, el ataque, calificado de éxito moderado, tuvo, sin embargo, un alto precio. Se estima que 133 de las 183 aeronaves fueron destruidas completamente y que solo quince pilotos regresaron a su unidad militar. Justo 
después de esta misión, la aviación alemana solicitó permiso para llevar a término auténticas operaciones suicidas, dadas las mínimas posibilidades de supervivencia. A los pilotos se les llamó los «hombres SO», del alemán Selbstopferung («autoinmolación»). De conformidad con investigaciones de la cadena de televisión pública alemana ZDF, entre el 16 y el 19 de abril de 1945 había todavía unos treinta y seis de estos pilotos en activo (Gunkel 2009). Herrmann no modificó su actitud radical ni siquiera después de la guerra. En el período de la posguerra vivió en Düsseldorf, donde ejerció como abogado, defendiendo a nazis y a negacionistas del Holocausto. Hizo, además, campaña a favor del Partido Nacionaldemócrata Alemán (NPD) y del partido Unión del Pueblo Alemán (DVU), ambos de extrema derecha. Herrmann falleció en Düsseldorf en noviembre de 2010. En la página de internet del NPD se halla una nota necrológica exaltando «la solemne ceremonia de uno de los últimos héroes alemanes» (NPD Bochum 2010). Herrmann «nunca se apartó de su valiente postura» y se sacrificó «por los derechos y la justa causa alemana, pese a toda la hostilidad que tuvo que afrontar [...]» (NPD Bochum 2010). El hecho de que no fuera sepultado con honores militares pone de manifiesto, según esta web, «la degradación del Estado alemán actual» (NPD Bochum 2010). Esto demuestra a las claras que Herrmann fue un nazi sin paliativos y lo siguió siendo hasta el final de su vida (véase también Gunkel 2009).

\section{Conclusión}

Espero haber mostrado algunos elementos de continuidad entre la Alemania del nacionalsocialismo y la posterior a su derrota en la Segunda Guerra Mundial en su trato con España y, en particular, con el régimen franquista. Durante la etapa de la República de Bonn, se recurre a todo tipo de contactos de la época del Tercer Reich. Además, diversas empresas involucradas anteriormente en negocios promovidos por la Alemania nazi fueron recuperadas con disimulo por la nueva Alemania occidental; se impulsaron en secreto, incluso, ambiciosos proyectos armamentísticos. Muchos militares alemanes que participaron de forma activa en la Guerra Civil (en las operaciones Fuego Mágico y Úrsula, y en la Legión Cóndor) ingresaron durante la posguerra en el Ejército o la Marina de la RFA. 
Este capítulo todavía no ha sido suficientemente estudiado por la historiografía alemana. Ocurre igual con la explotación económica de España por parte de la HISMA (con el beneplácito de Franco). Miembros de la Legión Cóndor como Werner Beumelburg o Hans-Joachim Herrmann, que indudablemente fueron nazis convencidos, formaron parte - sin que se les dirigiera reproche alguno - de la sociedad alemana de posguerra. El libro de Beumelburg Kampfum Spanien, publicado en 1939, fue objeto de reediciones acríticas incluso hasta el año 1980. Hans-Joachim Herrmann, inventor de los vuelos kamikazes, trabajó como abogado y, vinculado a círculos de extrema derecha, siguió negando el Holocausto.

Esto demuestra que en Alemania la confrontación crítica con el pasado nazi no fue siempre tan modélica como se afirma. Que la extrema derecha en Alemania desgraciadamente esté en auge hoy en día resulta menos sorprendente cuando nos damos cuenta de que nunca dejó de existir. Es un hecho que, poco después de la Segunda Guerra Mundial, se reanudaron viejas conexiones que no se habían cortado definitivamente. Los ejemplos aquí explicados confirman que aún queda mucho por hacer en materia de historia germano-española. Esta tarea, desde luego, requiere métodos rigurosos de estudio, y se deberían dedicar a ella conjuntamente investigadores de las áreas de historia y de estudios románicos. El conocimiento de los hechos históricos es un requisito previo para manejar responsablemente las memorias del pasado sin caer en la trampa de la desinformación propagandística. Todo esto nos recuerda dolorosamente la importancia del trabajo internacional sobre la memoria colectiva para el mantenimiento de la paz en la Europa contemporánea.

\section{Bibliografía}

Adam, Christian. 2010. Lesen unter Hitler. Autoren, Bestseller, Leser im Dritten Reich. Berlín: Galiani.

Aschmann, Birgit. 1999. «Treue Freunde...»? Westdeutschland und Spanien 1945-1963. Stuttgart: Steiner.

Bannasch, Bettina; Holm, Christiane, y Carl Freitag, ed. 2005. Erinnern und Erzählen. Der Spanische Bürgerkrieg in der deutschen und spanischen Literatur und in den Bildmedien. Tubinga: Narr. 
Bernecker, Walther L. 1986. «Das nationalsozialistische Spanien-Bild und Hitlers Eingreifen in den Spanischen Bürgerkrieg». Dentro Günther Schmigalle, ed., Der Spanische Bürgerkrieg. Literatur und Geschichte, 25-54. Fráncfort/M.: Vervuert.

. 2009. «Democracia y superación del pasado: sobre el retorno de la memoria histórica reprimida en España». Dentro Ignacio Olmos, Nikky Keilholz-Rüle y Helga Schneider, ed., La cultura de la memoria. La memoria histórica en España y en Alemania, 57-73. Madrid: Vervuert.

Briesemeister, Dietrich. 2004. Spanien aus deutscher Sicht. Deutsch-spanische Kulturbeziehungen gestern und heute, edición de Harald Wentzlaff-Eggebert. Tubinga: Niemeyer.

Chihaia, Matei, y Ursula Hennigfeld, ed. 2020. Guernica entre ícono y mito. Productividad y presencia de memorias colectivas. Madrid: Vervuert.

Collado Seidel, Carlos. 2012. «Spanischer Bürgerkrieg und Zweiter Weltkrieg: Wege der Auseinandersetzung». Dentro Jochen Mecke, Hubert Pöppel y Ralf Junkerjürgen, ed., Deutsche und Spanier - ein Kulturvergleich, 86-101. Bonn: Bundeszentrale für politische Bildung.

De la Hera Martínez, Jesús. 2002. La política cultural de Alemania en España en el período de entreguerras. Madrid: CSIC.

Deutsches U-Boot-Museum. Sin fecha. «Im spanischen Bürgerkrieg. Deutsche U-Boote im spanischen Bürgerkrieg». http://dubm.de/im-spanischen-buergerkrieg/. [Consulta: 13 enero 2020.]

Gunkel, Christoph. 2009. «Deutsche Kamikaze-Flieger. Himmelfahrtskommando für Hitler». Der Spiegel, 27 oct. https://www.spiegel.de/geschichte/deutsche-kamikazeflieger-a-948570.html. [Consulta: 13 enero 2020.]

Hausmann, Frank-Rutger. 2001. "Auch im Krieg schweigen die Musen nicht». Die Deutschen Wissenschaftlichen Institute im Zweiten Weltkrieg. Gotinga: Vandenhoeck \& Ruprecht.

Henkel, Peter. 2016. Die Düsseldorfer Konzentrationslager. Der Einsatz von KZ-Häftlingen in Düsseldorf zwischen 1942 und 1945. Düsseldorf: Droste.

Herzog, Bodo. 1991. «Piraten vor Malaga». Die Zeit, 29 nov. http://www.zeit.de/1991/49/ piraten-vor-malaga. [Consulta: 13 enero 2020.]

Hinrichs, Per. 2003. «Test für den Terror. Bisher unbekannte Dokumente belegen, dass Hitlers "Legion Condor" die baskische Stadt Guernica gezielt zerstörte - ebenso wie eine Reihe anderer spanischer Ortschaften». Spiegel Special 1: 33-35.

Inal, Benjamin. 2014. Gernika/Guernica als Erinnerungsort in der spanischsprachigen Literatur. Fráncfort/M.: Peter Lang.

Irving, David. 1965. «Unternehmen Armbrust. Der Kampf des britischen Geheimdienstes gegen Deutschlands Wunderwaffen». Der Spiegel, 17 nov.

Lehmann, Walter. 2006. Die Bundesrepublik und Franco-Spanien in den 5oer Jahren. NSVergangenheit als Bürde?. Múnich: Oldenbourg.

Lexikon der Wehrmacht. Sin fecha. «Spanienkreuz». http://www.lexikon-derwehrmacht.de/Orden/spk.html. [Consulta: 13 enero 2020.] 
NPD Bochum. 2010. «Gedenkfeier für Ritterkreuzträger Hajo Herrmann». http://npdbochum.de/1089/1089-2. [Consulta: 13 enero 2020.]

Olmos, Ignacio; Nikky Keilholz-Rühle, y Helga Schneider, ed. 20og. La cultura de la memoria. La memoria histórica en España y en Alemania. Madrid: Vervuert.

Oven, Wilfred von. 1978. Hitler und der Spanische Bürgerkrieg. Mission und Schicksal der Legion Condor. Tubinga: Grabert.

Rebok, Sandra. 2010. Traspasar fronteras. Un siglo de intercambio científico entre España y Alemania. Madrid: CSIC.

Reig Tapia, Alberto. 2012. «La pervivencia de los mitos franquistas». Dentro Ángel Viñas, ed., En el combate por la historia. La República, la Guerra Civil, el franquismo, 903920. Barcelona: Pasado y Presente.

Ruhl, Klaus-Jörg. 1975. Spanien im Zweiten Weltkrieg. Franco, die Falange und das «Dritte Reich». Hamburgo: Hoffmann und Campe.

Sanz Díaz, Carlos. 2006. España y la República Federal de Alemania (1949-1966). Política, Economía y Emigración entra la Guerra Fría y la Distensión. Madrid: Universidad Complutense.

Schmigalle, Günther. 1986. «Deutsche schreiben für Hitler und Franco. Vierzig bio-bibliographische Porträts». Dentro Günther Schmigalle, ed., Der Spanische Bürgerkrieg. Literatur und Geschichte, 197-243. Fráncfort/M.: Vervuert.

Schüler-Springorum, Stefanie. 2010. Krieg und Fliegen. Die Legion Condor im Spanischen Bürgerkrieg. Paderborn: Schöningh.

Thamm, Wolfgang. 2004. Hauptmann (W) und Ritterkreuzträger Heinz Schweizer. Feuerwerker und Sprengkommandoführer. Bissendorf: Biblio.

Thomàs, Joan Maria. 2019. «Introduction». Dentro Joan Maria Thomàs, Jon Irazabal Agirre y Ramon Arnabat Mata, ed., Bombs over Biscay, Barcelona and Dresden (1937-1945). From the Spanish Civil War to the Second World War, 7-9. Tarragona: Universitat Rovira i Virgili.

Whealey, Robert H. 2005. Hitler and Spain. The Nazi Role in the Spanish Civil War, 19361939. Louisville: University Press of Kentucky. 
